\title{
2 Die Nutzung der auf oder mittels der elektronischen Gesundheitskarte gespeicherten medizinischen Daten
}

\subsection{Vorbemerkungen}

Gemäß § 291a Abs. 1 SGB V soll die elektronische Gesundheitskarte der „Verbesserung von Wirtschaftlichkeit, Qualität und Transparenz der Behandlung“ dienen und erweitert damit die bestehende Krankenversicherungskarte. ${ }^{4}$ Der Einsatz der Karte und der auf und mittels ihr gespeicherten Daten zur medizinischen Forschung ist folglich allenfalls mittelbar (in dem Sinne, dass derartige Forschung ebenfalls der Verbesserung der Behandlung dient) angesprochen. Die elektronische Verarbeitung von Behandlungsinformationen, die mit der elektronischen Gesundheitskarte in weitem Umfang angestrebt und möglich wird, bietet jedoch zugleich immense Chancen für eine Forschung, die im nahen Umfeld der Behandlung stattfindet. ${ }^{5}$

Die Daten werden in leicht zu verarbeitender und auswertbarer Form vorliegen. Außerdem werden sowohl die elektronische Gesundheitskarte (zumindest optional) als auch der elektronische Heilberufsausweis über die Basisfunktionalitäten der elektronischen Signatur, Verschlüsselung und Authentifizierung verfügen, die als Sicher-

4 Zu den Erwartungen der Bürger s. Goetz, DuD 2010, 811ff.; allgemein zur elektronischen Gesundheitskarte s. z.B. Hornung 2005, v.a. 207ff., 246ff., 362ff.; ders. 2013, 51ff.; Bales/v. Schwanenflügel, NJW 2012, 2475; europäischer Überblick bei Reimer/Artmann/ Stroetmann, DuD 2013, 154ff.

5 Dementsprechend ist die Verbesserung der medizinischen Forschung (neben den Zielen der Verbesserung der Versorgung und der Kosteneinsparung durch Rationalisierungen) schon immer ein Ziel des Einsatzes von Informationstechnologie im Gesundheitswesen gewesen, so schon Schaefer 1979, 21; s.a. Lilie 1980, 26f.; Roßnagel/Wedde/Hammer/Pordesch 1990, 182. Die besonderen Chancen der elektronischen Patientendaten für die medizinische Forschung werden derzeit europaweit erkannt, s. Reimer/Artmann/Stroetmann, DuD 2013, 154. 
II Die Nutzung der elektronischen Gesundheitskarte und der Krankenversichertennummer im Forschungskontext

heitsdienste die Daten schützen und so im Forschungsumfeld Verwendung finden können.

Ursprünglich sollte die Einführung der elektronischen Gesundheitskarte laut $\$ 291 \mathrm{a}$ Abs. 1 SGB V „bis spätestens zum 1. Januar 2006“ erfolgen. Durch längere Diskussionen innerhalb der Selbstverwaltung um die technische Gestaltung, die organisatorische Umsetzung und die Finanzierung des Großprojekts wurde dieses immer wieder verzögert. Der Gesetzgeber griff daraufhin zu der in $\mathbb{} 4$ Abs. 6 SGB V vorgesehenen Drohung, den Krankenkassen die Verwaltungsausgaben um zwei Prozent zu kürzen, sofern diese nicht bis zum 31. Dezember 2011 an mindestens zehn Prozent ihrer Versicherten elektronische Gesundheitskarten ausgegeben hatten, sowie die Verwaltungsausgaben für das Jahr 2013 nicht zu erhöhen, falls die Ausgabequote bis zum 31. Dezember 2012 nicht mindestens 70 Prozent betrüge. ${ }^{6}$ Dies zeigte Wirkung: Seit 2014 besitzen 97 Prozent der 70 Millionen gesetzlich Versicherten eine elektronische Gesundheitskarte, ${ }^{7}$ auch wenn diese in der Praxis bei weitem noch nicht zu den Funktionalitäten einsetzbar ist, die der Gesetzgeber vorgesehen hat. Mit der Nutzung von medizinischen Anwendungen ist erst ab dem Jahre $2018 \mathrm{zu}$ rechnen. ${ }^{8}$

Das Fünfte Buch des Sozialgesetzbuches (SCB V) enthält seit nunmehr über zehn Jahren detaillierte Vorgaben für die Funktionen der elektronischen Gesundheitskarte, die auf und mittels ihr gespeicherten Daten, die zugelassenen Verwendungen und die Zugriffsberechtigungen der verschiedenen Beteiligten im Gesundheitswesen. Die Umsetzung dieser Vorgaben in technische Verfahren und Zugriffssicherungen vorausgesetzt, ermöglicht dies bereits vor der Einführung der entsprechenden Funktionalitäten die Analyse der rechtlichen Zulässigkeit und faktischen Umsetzbarkeit des Einsatzes der elektronischen Gesundheitskarte im Kontext der medizinischen Forschung.

Der Gesetzgeber hat bei der Regulierung der Telematik-Infrastruktur - deren Herzstück die elektronische Gesundheitskarte werden wird, sobald sie zu den ihr zugedachten Funktionen einsetzbar ist - den Fokus auf Versorgung der Patienten gelegt und die Frage der medizinischen Forschung nicht explizit geregelt. Außerdem wurden - zutreffender Weise - erhebliche datenschutzrechtliche Risiken identifiziert, die aus der zunehmenden elektronischen Datenerhebung, -verarbeitung und -nutzung, insbesondere der erleichterten Datenzusammenführung, resultieren. ${ }^{9}$ Deshalb wurden starke Verwendungsbeschränkungen vorgesehen, die im Folgenden zu untersuchen sind. Dabei wird mit der vorliegenden Analyse Neuland beschritten, da soweit ersichtlich bislang keine Literatur zur Frage der Nutzung der elektronischen Gesundheitskarte im Bereich der medizinischen Forschung existiert. ${ }^{10}$

Zwar hat das Bundessozialgericht im Jahre 2008 die allgemeinen Regelungen des Datenschutzes, die die Datenübermittlung bei Vorliegen einer Einwilligungserklärung des Betroffenen erlauben, sehr pauschal „im Geltungsbereich des Sozialgesetz-

\footnotetext{
S. Hornung 2013, $52 f$.

S. Basil 2014.

S. Basil 2014.

In Tschechien wurde das parallele Projekt einer elektronischen Gesundheitskarte nach zehn Jahren unter anderem wegen Datenschutzbedenken sogar wieder gestoppt (RDG 2012, 165). Der im Jahre 2013 aufgeflogene Verkauf von Patientendaten durch ein Apothekenrechenzentrum an ein Marktforschungsinstitut zeigt das bestehende datenschutzrechtliche Risiko auch in Deutschland schon ohne die geplante Karte (ZD-Aktuell 2013, 03699 sowie Weichert, DuD 2013, 130).

10 Auszüge dieses Abschnitts flossen bereits ein in Roßnagel/Hornung, MedR 2008, 538ff.; s.a. Hornung 2013, $67 f$.
} 
2 Die Nutzung der auf oder mittels der elektronischen Gesundheitskarte gespeicherten medizinischen Daten

buches “ für unanwendbar erklärt, weil der Gesetzgeber detaillierte bereichsspezifische Regelungen in das Sozialgesetzbuch aufgenommen habe. ${ }^{11}$ Diese Schlussfolgerung ist jedoch nicht durch die Argumentation des Gerichts gedeckt, die stringent ausschließlich für die krankenversicherungsrechtlichen Vorschriften zum Sozialdatenschutz in den $\$ \mathbb{S} 284 \mathrm{ff}$. SGB V den abschließenden Charakter nachgewiesen hat. ${ }^{12}$ Für andere Bereiche des Sozialgesetzbuchs wäre dieser Nachweis noch zu führen, denn die Leistungsdaten zur Abrechnung sind anders zu behandeln als die medizinischen Behandlungs- und Versorgungsdaten, die gerade nicht unter die $\mathbb{s} 284 \mathrm{ff}$. SGB V fallen. Des Weiteren sind medizinische Forschungsdaten nicht zwingend Sozialdaten im Sinne des $\$ 67$ Abs. 1 Satz 1 SGB X. Für andere Daten kann jedoch nicht von einer umfassenden und abschließenden Regelung des Sozialgesetzbuches ausgegangen werden, sodass die subsidiäre Anwendbarkeit der Ermächtigungsvorschriften des Bundesdatenschutzgesetzes oder der Landesdatenschutzgesetze nicht von vornherein ausgeschlossen sind. ${ }^{13}$ Daher ist im Folgenden die Anwendbarkeit von Einwilligungsregeln anhand der Normen des konkreten Regelungsbereiches - hier der Vorschriften über die Datenverarbeitung auf und mittels der elektronischen Gesundheitskarte - zu überprüfen.

\subsection{Die derzeitige Zulässigkeit der Verwendung der auf oder mittels der elektronischen Gesundheitskarte gespeicherten Daten zu Forschungszwecken}

Im geltenden Recht findet sich keine ausdrückliche Bestimmung zur Zulässigkeit der Verwendung der auf oder mittels der elektronischen Gesundheitskarte gespeicherten Daten zu Zwecken der medizinischen Forschung; forschende Tätigkeit wird lediglich im Zusammenhang mit den Forschungs- und Entwicklungstätigkeiten für die Telematik-Infrastruktur erwähnt. ${ }^{14}$ Im Fünften Buch des Sozialgesetzbuches wurde allerdings für die elektronische Gesundheitskarte eine Vielzahl von detaillierten Verwendungsbestimmungen und -beschränkungen geregelt. ${ }^{15}$ Diese Normen erfassen - und beschränken - auch die Verwendung der Daten zu Zwecken, die nicht im Gesetz geregelt sind. ${ }^{16}$

\subsubsection{Zugriff von Forschern auf vorhandene Behandlungsdaten}

\section{Dürfen Forscher mit Einverständnis des Patienten Zugriff auf Behandlungsdaten bekommen?}

\footnotetext{
11 BSGE 102, 134; s. Z.B. Brisch/Laue, CR 2009, 265ff.; Kühling/Seidel, GesR 2010, 231ff.; Leisner, NZS 2010, 129ff.; Schneider, VSSR 2009, $381 \mathrm{ff}$.

12 S. hierzu Roßnagel/Hornung/Jandt 2009, Teil I, Kap. 1.1.

13 S. zur Entscheidung des Bundessozialgerichts insoweit ausführlich Roßnagel/Hornung/Jandt 2009, Teil I, Kap. 1.1.

14 Vgl. \ 291b Abs. 5 und 6 SGB V; näher Hornung, in: Hänlein/Kruse/Schuler, \ 291b Rn. 5, \$ 291a Rn. 21 m.w.N.

15 S. ausführlich Hornung 2005, 207ff., 362ff. et passim; Bales/Dierks/Holland/Müller 2007, 39ff.; s.a. Borchers 2008, 93ff.

16 Zu den Regulierungsfragen der Telematik-Infrastruktur s. Pitschas, NZS 2009, $177 \mathrm{ff}$.
} 
II Die Nutzung der elektronischen Gesundheitskarte und der Krankenversichertennummer im Forschungskontext

\subsubsection{Verwendungsregel in $§ 291$ SGB V}

\$ 291 Abs. 1 Satz 3 SGB V enthält eine Verwendungsregel für die aktuelle Krankenversichertenkarte, die zur elektronischen Gesundheitskarte ausgebaut wird. Dort heißt es:

„Sie [die Krankenversichertenkarte] darf vorbehaltlich \$2 291a nur für den Nachweis der Berechtigung zur Inanspruchnahme von Leistungen im Rahmen der vertragsärztlichen Versorgung sowie für die Abrechnung mit den Leistungserbringern verwendet werden."

Da $\$ 291 a$ SCB V nicht die aktuelle Krankenversichertenkarte, sondern die elektronische Gesundheitskarte betrifft, ergibt sich daraus unmissverständlich, dass erstere nicht zu Forschungszwecken eingesetzt werden darf. Aufgrund des ausdrücklichen Vorbehalts in $\$ 291$ Abs. 1 Satz 3 SGB V ist die Frage für die elektronische Gesundheits-

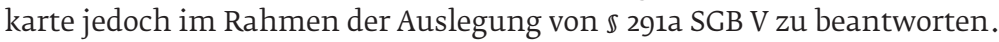

\subsubsection{Auslegung von $\$ 291 \mathrm{a}$ SGB V}

Die gesetzlich vorgesehenen Funktionen der elektronischen Gesundheitskarte sind in $\$ 291$ abs. 2 (Pflichtanwendungen) und Abs. 3 (freiwillige Anwendungen) SCB V geregelt. ${ }^{17}$ Die drei verpflichtenden Teile ( $\$ 291 a$ Abs. 2 Satz 1 SGB V) sind die Speicherung der Versicherungsstammdaten, die Übermittlung des elektronischen Rezepts sowie die Ablage des Berechtigungsnachweises zur Inanspruchnahme von Leistungen in den Mitgliedstaaten der Europäischen Union. $\$ 291 a$ Abs. 3 Satz 1 SGB V enthält demgegenüber diejenigen Anwendungen, zu deren Ausführung die elektronische Gesundheitskarte zwar in der Lage sein muss, über deren Einsatz der Inhaber jedoch selbst entscheiden kann. ${ }^{18}$ Im Einzelnen sind dies die medizinischen Notfalldaten, ${ }^{19}$ der elektronische Arztbrief (Befunde, Diagnosen, Therapieempfehlungen und Behandlungsberichte für einen einrichtungsübergreifenden Behandlungsfall), die elektronische Patientenakte (ebendiese Angaben sowie Impfungen, jedoch fallübergreifend), ${ }^{20}$ die Daten zur Prüfung der Arzneimitteltherapiesicherheit, ${ }^{21}$ vom Patienten selbst oder für ihn zur Verfügung gestellte Daten, ${ }^{22}$ Daten über in Anspruch genom-

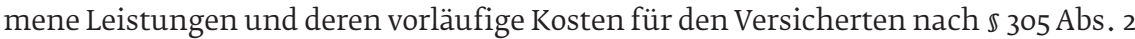
SGB V („Patientenquittung“), Erklärungen des Patienten zur Organ- und Gewebespende (alternativ auch Hinweise auf das Vorhandensein und den Aufbewahrungsort

17 S. zum Folgenden näher Hornung 2005, 6off.; ders. 2004, 226ff.; Hornung/Goetz/Goldschmidt, WI 2005, 171ff.; Bales/Dierks/Holland/Müller 2007, 82ff.

18 Die Einwilligung bedarf nach umstrittener, aber zutreffender Ansicht gemäß § 291a Abs. 3 Satz 4 SGB V i.V.m. \4a Abs. 1 Satz 3 BDSG der Schriftform, s. Hornung, in: Hänlein/Kruse/Schuler, \$ 291a Rn. 9 auch zur anderen Ansicht.

19 In Anlehnung an den international standardisierten Notfalldatensatz ISO 21549-3. Das Gesetz enthält keine Angaben darüber, welche Informationen im Einzelnen gemeint sind und ließe folglich auch patientenspezifische Notfalldaten zu. Hierfür kämen chronische Organleiden, Dialyseinformationen, Allergien und Arzneimittelunverträglichkeiten in Betracht, vgl. Weichert, DuD 2004, 391, 396.

20 Zur Rechtslage bezüglich des elektronischen Arztbriefs und der elektronischen Patientenakte vor Inkrafttreten des GKV-Modernisierungsgesetztes s. Dierks/Nitz/Grau 2003, 109f., 113ff.; s.a. Grätzel v. Grätz 2004, 62ff.

21 Dieser Terminus wurde durch das Gesetz zur Vereinfachung der Verwaltungsverfahren im Sozialrecht (BGBI. I 2005, 818) an die Stelle von „Arzneimitteldokumentation“ gesetzt und soll neben den verordneten Arzneimitteln auch akute und chronische Erkrankungen umfassen, s. die Gesetzesbegründung, BT-Drs. 15/4228, 28.

22 Die Gesetzesbegründung nennt beispielhaft Verlaufsprotokolle bei chronischen Krankheiten und Patientenverfügungen (BT-Drs. 15/1525, 145), die Begründung des ersten Entwurfs auch den Organspendeausweis (BT-Drs. 15/1170, 123). 
2 Die Nutzung der auf oder mittels der elektronischen Gesundheitskarte gespeicherten medizinischen Daten

derartiger Erklärungen) sowie Hinweise auf das Vorhandensein und den Aufbewahrungsort von Vorsorgevollmachten oder Patientenverfügungen nach $\mathbb{} 1901$ a BCB. ${ }^{23}$

Weitere Funktionen und Verwendungen der elektronischen Gesundheitskarte sind nicht gesetzlich geregelt. Das bedeutet aber nicht, dass diese per se zulässig wären. Der Gesetzgeber hat sich vielmehr dafür entschieden, die Datenverwendung stark einzuschränken. Dies ist insbesondere durch $\$ 291 \mathrm{a}$ Abs. 8 SGB V geschehen. Die Auslegung dieser Norm ist das Hauptproblem der Frage der Zulässigkeit des Einsatzes der elektronischen Gesundheitskarte zu Forschungszwecken nach geltendem Recht. ${ }^{24}$ Die Vorschrift lautet wie folgt:

„Vom Inhaber der Karte darf nicht verlangt werden, den Zugriff auf Daten nach Absatz 2 Satz 1 Nr. 1 oder Absatz 3 Satz 1 anderen als den in Absatz 4 Satz 1 und Absatz 5a Satz 1 genannten Personen oder zu anderen Zwecken als denen der Versorgung der Versicherten, einschließlich der Abrechnung der zum Zwecke der Versorgung erbrachten Leistungen, zu gestatten; mit ihnen darf nicht vereinbart werden, Derartiges zu gestatten. Sie dürfen nicht bevorzugt oder benachteiligt werden, weil sie einen Zugriffbewirkt oder verweigert haben."

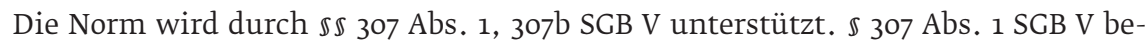
stimmt:

„Ordnungswidrig handelt, wer entgegen $\$ 291 a$ Abs. 8 Satz 1 eine dort genannte Gestattung verlangt oder mit dem Inhaber der Karte eine solche Gestattung vereinbart."

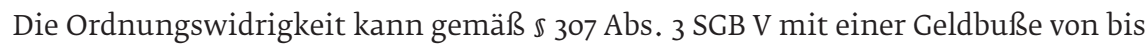
zu 50.00o Euro geahndet werden. $\$ 307 \mathrm{~b}$ Abs. 1 SGB V lautet:

„Mit Freiheitsstrafe bis zu einem Jahr oder mit Celdstrafe wird bestraft, wer entgegen \$2 $291 a$ Abs. 4 Satz 1 oder Absatz 5 a Satz 1 erster Halbsatz oder Satz 2 auf dort genannte Daten zugreift."

Handelt der Täter gegen Entgelt oder in der Absicht, sich oder einen anderen zu be-

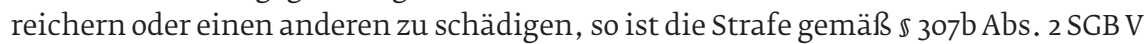
Freiheitsstrafe bis zu drei Jahren oder Geldstrafe.

Zu klären ist, ob \$ $291 \mathrm{a}$ Abs. 8 SGB V auch die Nutzung der auf oder mittels der elektronischen Gesundheitskarte gespeicherten Daten zu Forschungszwecken erfasst und verbietet. Diese Frage ist entsprechend den anerkannten Methoden der Auslegung ${ }^{25}$ nach Wortlaut, Systematik, Gesetzgebungsgeschichte sowie Sinn und Zweck der Norm zu beantworten.

Der Wortlaut von $\$ 291$ a Abs. 8 Satz 1, 1. Halbsatz SGB V ist nicht einschlägig, wenn und solange diejenigen Personen, die für die forschenden Institutionen die Einwilligung einholen, von den Patienten diese nicht „verlangen“. Anwendbar ist aber $\$ 291 \mathrm{a}$ Abs. 8 Satz 1, 2. Halbsatz SGB V. Dieser schließt grundsätzlich jede Vereinbarung über eine Gestattung des Zugriffs zu anderen Zwecken als denen der Versorgung aus. Man könnte höchstens vertreten, es liege keine Vereinbarung vor, wenn ein Arzt den Patienten über die Möglichkeit der Teilnahme an einer Studie informiert und dieser

23 Die Möglichkeiten der Speicherung von Informationen zu Organ- und Gewebespende sowie zu Vorsorgevollmachten und Patientenverfügungen wurden durch das Gesetz zur Regelung der Entscheidungslösung im Transplantationsgesetz vom 12.7.2012, BGBl. I, 1504, eingeführt.

24 S. zu diesem bereits Hornung 2005, 237ff.; ders. 2004, 233; im hiesigen Kontext Roßnagel/Hornung, MedR 2008, 539f.; s.a. Hornung $2013,62 f$.

25 Dazu z.B. Larenz/Canaris 1995, 133ff.; Röhl/Röhl 2008, 628ff.; Rüthers/Fischer/Birk 2013, Rn. 696ff. 
II Die Nutzung der elektronischen Gesundheitskarte und der Krankenversichertennummer im Forschungskontext

dann direkt einwilligt. Dies erscheint jedoch schon mit dem Wortlaut kaum vereinbar und wird auch Sinn und Zweck der Norm nicht gerecht (s.u.).

Die systematische Auslegung ergibt keine weiterführenden Ergebnisse.

Die Gesetzgebungsgeschichte birgt keine Erkenntnisse zur Frage der Verwendung der Daten für die medizinische Forschung. Sowohl im ersten Entwurf der Fraktionen SPD und BÜNDNIS 9o/DIE GRÜNEN ${ }^{26}$ als auch in der letztlichen Begründung des Gesetzes, die auch von der Fraktion CDU/CSU mitgetragen wurde, ${ }^{27}$ heißt es zum heutigen $\$ 291 \mathrm{a}$ Abs. 8 SGB V lapidar:

„Die Regelung dient dem Schutz der Gesundheitskarte vor missbräuchlicher Verwendung.“

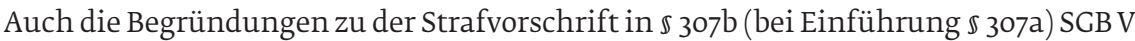
und den Ordnungswidrigkeiten in $\$ 307$ Abs. 1 SGB V erwähnen die Forschung nicht. ${ }^{28}$ In der Begründung zu $\$ 307 \mathrm{Abs}$. 1 SGB V heißt es:

„Es werden bereits Handlungen erfasst, die im Vorfeld eines verbotenen Zugriffs aufdie Daten anzusiedeln sind. Es soll insbesondere verhindert werden, dass in Situationen, in denen die Karteninhaber einem besonderen Druck ausgesetzt sind, die Einwilligung der Karteninhaber zum Zugriff aufihre Daten verlangt oder eine entsprechende Vereinbarung mit ihnen getroffen wird."

Diese Formulierung führt für die Frage der Mitnutzung von Daten für Forschungszwecke letztlich nicht zu eindeutigen Ergebnissen. Einerseits wird sich der Patient meist bei seinem behandelnden Arzt befinden, dem er vertraut. Andererseits besteht durchaus eine Situation, in der das primäre Interesse an der Genesung so stark ist, dass die Gefahr einer Drucksituation besteht.

Ihrem Sinn und Zweck nach (teleologische Auslegung) sollen $\$ 291 \mathrm{~A}$ Abs. 8 und $\$ 307$ Abs. 1 SGB V vor Einflussnahmen auf den Versicherten schützen. ${ }^{29}$ Das Projekt der elektronischen Gesundheitskarte basiert auf dem erweiterten, erleichterten und schnelleren Zugang zu Gesundheitsdaten. Dies ist nützlich und sinnvoll, solange diese Daten zum Zwecke der Gesundheitsvorsorge verwendet werden. Gleichzeitig steigt aber die Gefahr von Begehrlichkeiten durch Personen oder Institutionen, zu denen der Versicherte in sozialen Abhängigkeitsverhältnissen steht (Arbeitgeber, Versicherungen etc.).

Der Patient ist insoweit in einer zwiespältigen Position. ${ }^{30}$ Je stärker seine Rolle im Gesamtgefüge des Informationsflusses ist, desto mehr wird seine Stellung als eigentliches Subjekt der Datenverarbeitung im Gesundheitswesen betont und seine informationelle Selbstbestimmung gestärkt. Eine weitgehende Entscheidungsbefugnis des Einzelnen hat jedoch zur Folge, dass seine Entscheidung über eine Datenfreigabe von seinem sozialen Umfeld beeinflusst werden kann. Dieses Risiko besteht zwar bereits heute, wird aber durch die leichtere Verfügbarkeit der Daten verschärft. So bietet die elektronische Gesundheitskarte, konsequent angewendet, den Zugang zu einer elektronischen Patientenakte mit allen oder allen wesentlichen Informationen über die gesamte Krankengeschichte des Versicherten, wenn er es wünscht. Im heu-

26 BT-Drs. 15/1170, 123

27 BT-Drs. 15/1525, 145.

28 BT-Drs. 15/1170, 128; BT-Drs. 15/1525, 151

29 S. zum Folgenden Hornung 2005, $238 f$.

30 S. Hornung 2005, $238 f$. 
tigen System gibt es eine derartige Datensammlung nicht, und die Zusammenführung kann dem Versicherten auch unter sozialem Druck kaum abgenötigt werden. Besteht dagegen eine elektronische Patientenakte, so kann er bei einem beliebigen Arzt eine Art „Gesundheitsauszug“ über seine Krankengeschichte und den aktuellen Gesundheitszustand erhalten. Diese Vereinfachung erhöht das Risiko, dass der Versicherte psychischem oder materiellem sozialen Druck ausgesetzt wird und diesem nachgibt. Dies könnte im Ergebnis das Vertrauensverhältnis zwischen Arzt und Patient beschädigen, dessen individuelle und gesellschaftliche Bedeutung sowohl das Bundesverfassungsgericht als auch der Europäische Gerichtshof für Menschenrechte betont haben. ${ }^{31}$

Die genannten Risiken sind bei der Einwilligung zu medizinischen Forschungszwecken nicht in gleichem Maße gegeben. Dies spräche dafür, eine solche bereits de lege lata zuzulassen. Der Gesetzgeber hat sich aber vor dem Hintergrund der genannten Risiken zu einer rigorosen Lösung, nämlich der absoluten Beschränkung der Verwendung der Behandlungsdaten auf Versorgungszwecke, entschieden. Auch wenn sich in den Gesetzesmaterialien keine Erwägungen zur medizinischen Forschung finden lassen, muss man doch davon ausgehen, dass dem Gesetzgeber bewusst war, dass ein derartiger rigider Ausschluss der Datenverwendung auch nach Einwilligung des Patienten in Einzelfällen sinnvolle Tätigkeiten und Verwendungszwecke ausschließen würde.

Denkbar wäre schlussendlich eine teleologische Reduktion der Norm, zu deren Begründung auch die Forschungsfreiheit (Art. 5 Abs. 3 CG) herangezogen werden könnte. Absolute Grenze einer solchen Reduktion - wie auch der juristischen Auslegung insgesamt - ist jedoch der noch mögliche Wortsinn einer Norm. ${ }^{32}$ Der Wortlaut ist, wie oben dargelegt, eindeutig und schließt jede Verwendung der Behandlungsdaten zu anderen Zwecken als denen der Versorgung aus. Im Ergebnis sind folglich die Daten nach $\$ 291$ A Abs. 2 Satz 1 Nr. 1 und Abs. 3 Satz 1 SGB V für die medizinische Forschung nicht nutzbar.

Auch im Kontext der elektronischen Gesundheitskarte bleibt dabei das Problem bestehen, dass im Einzelfall gegebenenfalls die Entscheidung schwierig sein kann, ob (noch) eine Versorgung des Patienten oder (schon) medizinische Forschung vorliegt. Dieses Abgrenzungsproblem kann für die Praxis misslich sein, ist aber aus Rechtsgründen unvermeidbar. Wenn sich in einem bestimmten Kontext Behandlungs- und Forschungszweck mischen, ließe sich eine Abgrenzung nach Haupt- und Nebenzweck vornehmen. Die auf oder mittels der elektronischen Gesundheitskarte gespeicherten Daten könnten dann eventuell zulässigerweise genutzt werden, wenn der Schwerpunkt der Tätigkeit eindeutig behandelnder Art ist und sich das Forschungsergebnis

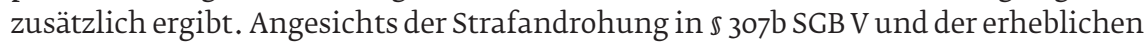
Bußgelddrohung von bis zu 50.000 Euro in $\$ 307$ SGB V kann aber für die Praxis nur davon abgeraten werden, sich in derartige Grenzbereiche zu begeben.

31 S. BVerfGE 32, 370 (380) und EGMR, Z ./. Finnland, Urteil v. 25.2.1997 (abrufbar unter: http://www.echr.coe.int/Eng/Judgments. htm), Abs. 95

32 S. BVerfGE 71, 115; 87, 224; 90, 263 (275); Zippelius 2012, 39; Rüthers/Fischer/Birk 2013, Rn. 736. 
II Die Nutzung der elektronischen Gesundheitskarte und der Krankenversichertennummer im Forschungskontext

\title{
2.2.1.3 Zwischenergebnis
}

Vor diesem Hintergrund ist die Frage zu Kapitel II.2.2.1 nach geltendem Recht wie folgt zu beantworten: $\mathbb{S} 291 \mathrm{a}$ Abs. 8 SGB V schließt eine rechtfertigende Einwilligung (um eine solche handelt es sich im Rechtssinn, da es um die vorherige Zustimmung geht) des Patienten in einen Zugriff auf die auf oder mittels der elektronischen Gesundheitskarte gespeicherten Daten jenseits der genannten Zwecke aus. Erfolgt der Zugriff ohne Einwilligung, liegt gemäß $\mathbb{3} 307 \mathrm{~b}$ SGB V eine Straftat vor. Auch wenn eine Einwilligung vorliegt - und diese ordnungsgemäß ist, das heißt informiert und freiwillig gegeben sowie schriftlich erteilt wurde - bleibt der Zugriff durch Forscher verboten und stellt gemäß $\mathbb{3} 307$ Abs. 1 SGB V eine Ordnungswidrigkeit dar. Dieses Ergebnis deckt sich inhaltlich mit der Feststellung des Bundessozialgerichtes, dass die abschließenden Regelungen des Sozialdatenschutzes im Fünften Buch des Sozialgesetzbuches den Rückgriff auf die allgemeinen Regeln einer datenschutzrechtlichen Einwilligung ausschließen. ${ }^{33}$

\subsubsection{Speicherung zusätzlicher Forschungsdaten auf der elektronischen Gesundheitskarte}

\author{
Dürfen zusätzliche Forschungsdaten für Zwecke der Forschung mit Einverständ- \\ nis des Patienten auf der elektronischen Gesundheitskarte gespeichert und ver- \\ waltet werden?
}

Eine zweite Einsatzmöglichkeit der elektronischen Gesundheitskarte zu Forschungszwecken könnte darin bestehen, nicht die zu Behandlungszwecken gespeicherten Daten zu nutzen, sondern zusätzliche Forschungsdaten auf oder mittels der Karte zu speichern. Diese Variante würde von der Entscheidung des Bundessozialgerichtes ${ }^{34}$ zumindest bei einer engen Auslegung des Urteils nicht erfasst, weil es sich nicht um Patientendaten im Geltungsbereich des Fünften Buches des Sozialgesetzbuches handelt.

\subsubsection{Zusätzliche Daten im Rahmen von § 291a SGB V}

『 291a SGB V lässt vom Wortlaut her grundsätzlich zwei Möglichkeiten zusätzlicher Datenspeicherungen auf der elektronischen Gesundheitskarte zu:

- Zum einen könnten diese „von Versicherten selbst oder für sie zur Verfügung gestellte Daten“ im Sinne von $\mathbb{S}$ 291a Abs. 3 Satz 1 Nr. 5 SGB V sein. Die Norm sieht ausdrücklich die Möglichkeit vor, dass der Karteninhaber selbst weitere Daten zur Speicherung und Nutzung zur Verfügung stellt oder Dritte ermächtigt, derartige Daten zur Verfügung zu stellen. Nach der Gesetzesbegründung kommen hierfür etwa Verlaufsprotokolle bei chronischen Krankheiten oder

33 BSGE 102, 134; s.o. Kap. II.2.1.

34 BSGE 102, 134; s.o. Kap. II.2.1. 
2 Die Nutzung der auf oder mittels der elektronischen Gesundheitskarte gespeicherten medizinischen Daten

Patientenverfügungen in Betracht; 35 die Begründung des ersten Entwurfs nennt (zugriffstechnisch problematisch) auch den Organspendeausweis. ${ }^{36}$ Organ- und Gewebespenden sowie Versorgungsvollmachten und Patientenverfügungen sind seit dem Jahre 2012 allerdings gesondert in $\mathbb{\$} 291 \mathrm{a}$ Abs. 3 Satz 1 Nr. 7 bis Nr. 9 SGB V geregelt.

- Zum anderen könnte erwogen werden, die Infrastruktur der elektronischen Gesundheitskarte und der mit ihr interagierenden Telematik-Infrastruktur parallel zu den in $\mathbb{2} 291 \mathrm{a}$ Abs. 2 und Abs. 3 SGB V genannten Verarbeitungszwecken auch für andere Zwecke zu nutzen. Weder $\mathbb{2} 291 \mathrm{a} A$ bs. 2 noch Abs. 3 SGB V sind von ihrem Wortlaut her abschließend. Beide verpflichten dazu, die elektronische Gesundheitskarte mit bestimmten Funktionen auszustatten, schließen aber weitere Funktionen nicht aus. $\$ 291$ a Abs. 3 SGB V bestimmt sogar ausdrücklich, die elektronische Gesundheitskarte müsse „insbesondere“ das Erheben, Verarbeiten und Nutzen der dann folgend genannten Datenkategorien ermöglichen. ${ }^{37}$

Beide Optionen können allerdings nur unter den weiteren Einschränkungen von \$2 291a Abs. 4, 5 und 8 SGB V wahrgenommen werden. Das bedeutet zunächst, dass gemäß $\$ 291 a$ Abs. 5 Satz 1 SGB V die Einwilligung der Karteninhaber einzuholen ist. Außerdem dürfen auf die Daten des Patientenfachs (die erste oben genannte Option) gemäß $\$ 291 a$ Abs. 4 Satz 1 Nr. 2 SGB V nur

„a) Ärzte,

b) Zahnärzte,

c) Apotheker, Apothekerassistenten, Pharmazieingenieure, Apothekenassistenten,

d) Personen, die

aa) bei den unter Buchstabe a bisc Genannten oder

bb) in einem Krankenhaus

als berufsmäßige Gehilfen oder zur Vorbereitung aufden Beruftätig sind, soweit dies im Rahmen der von ihnen zulässigerweise zu erledigenden Tätigkeiten erforderlich ist und der Zugriff unter Aufsicht der in Buchstabe a bis c Genannten erfolgt,

e) nach Absatz 3 Satz 1 Nr. 1 in Notfällen auch Angehörige eines anderen Heilberufs, der für die Berufsausübung oder die Führung der Berufsbezeichnung eine staatlich geregelte Ausbildungerfordert,

f) Psychotherapeuten“"

zugreifen. Mit anderen Worten wäre etwa eine Forschung durch Ärzte unproblematisch, durch andere, in $\mathbb{2} 291$ Abs. 4 Satz 1 Nr. 2 SGB V nicht genannte Personen jedoch nicht möglich.

35 BT-Drs. 15/1525, 145.

36 BT-Drs. 15/1170, 123; zur Problematik der ursprünglich missglückten Regelung der technischen Absicherung s. Hornung 2004, 232f.; ders. 2005, 226f.; dies ist nunmehr durch die detaillierten Vorgaben in \$291a Abs. 5a SGB V (eingeführt durch das Gesetz zur Regelung der Entscheidungslösung im Transplantationsgesetz vom 12.7.2012, BGBI. I, 1504) behoben.

37 S. Hornung 2005, 211f.; Bales/Dierks/Holland/Müller 2007, 86. 
II Die Nutzung der elektronischen Gesundheitskarte und der Krankenversichertennummer im Forschungskontext

\subsubsection{Verwendungsbeschränkung für die zusätzlichen Daten?}

Das größte Problem der Speicherung zusätzlicher Daten - in beiden oben genannten Fällen - ist allerdings die Verwendungsbeschränkung in $₫$ 291a Abs. 8 SGB V. Wenn diese auch die „unbenannten“ Beispiele der Datenspeicherung im Patientenfach und neben den Funktionen der elektronischen Gesundheitskarte betrifft, ist eine Nutzung zu Forschungszwecken nicht möglich.

Im Grundsatz erscheint es zwar denkbar zu argumentieren, dass $\mathbb{} 291 \mathrm{a}$ Abs. 8 SGB V sich auf die benannten Beispiele in $\$ 291$ a Abs. 2 und Abs. 3 SGB V beschränkt. Im Ergebnis ist eine solche Auslegung jedoch abzulehnen.

Für eine Nutzung der elektronischen Gesundheitskarte zu Forschungszwecken ohne die Beschränkung in $\$ 291$ Abs. 8 SGB V ließe sich zunächst die Widersprüchlichkeit der geltenden Regelung vorbringen: Die oben zitierte Gesetzesbegründung zu $\mathbb{2 9 1 a}$ Abs. 3 Satz 1 Nr. 5 SGB V zeigt, dass der Gesetzgeber ursprünglich für das Patientenfach gerade Anwendungen angedacht hatte, die wie Patientenverfügung und Organspendeausweis nicht der medizinischen Versorgung dienen, sondern darüber hinausgehen. Es wäre widersinnig, wenn $₫ 291$ A Abs. 8 SGB V absolut gelten und damit die Nutzung der so gespeicherten Daten verbieten würde. Hinsichtlich weiterer Funktionen neben dem Patientenfach ließe sich vertreten, dass $\mathbb{2} 291 \mathrm{~A} A$ As. 8 SGB V nur die bereits geregelten Anwendungen betrifft.

Auch aus einem zweiten Grund erscheint eine absolute Reichweite von $\mathbb{2} 291 \mathrm{a}$ Abs. 8 SCB V wenig sinnvoll: Patienten konnten schon immer in die Verwendung ihrer Behandlungsdaten zu Forschungszwecken einwilligen, wenn diese bei dem behandelnden Arzt gespeichert sind. Hieran hat sich durch die Regelungen zur elektronischen Gesundheitskarte nichts geändert. Dem Patienten wird durch $\$ 291 a$ Abs. 8 SGB V auch nicht verwehrt, in die Verwendung von Daten zu Forschungszwecken einzuwilligen, die zuvor mittels der elektronischen Gesundheitskarte - etwa als elektronischer Arztbrief nach $\mathbb{2} 291$ Abs. 3 Satz 1 Nr. 2 SGB V - übermittelt wurden. Ein anderes Ergebnis würde die medizinische Forschung zumindest ab dem Zeitpunkt massiv beeinträchtigen, in dem die Übermittlung von Behandlungsinformationen standardmäßig oder überwiegend unter Verwendung der elektronischen Gesundheitskarte erfolgt. Letztlich dürfte es für den Patienten wenig Unterschied machen, ob der behandelnde Arzt die Daten zunächst zu Behandlungszwecken aus der elektronischen Gesundheitskarte ausliest und dann auf Basis einer gültigen Einwilligung in seinem System zu Forschungszwecken weiterverwendet, oder ob die Daten bereits auf Basis einer derartigen Einwilligung direkt zu Forschungszwecken ausgelesen werden. $\$ 291 a$ Abs. 8 SGB V würde allenfalls Umgehungen erfassen, etwa, wenn Daten nur zum Schein zu Behandlungszwecken ausgelesen werden, um im Anschluss daran in die Verwendung zu anderen Zwecken einwilligen zu können. Hier werden aber in der Praxis erhebliche Abgrenzungsprobleme entstehen.

Schlussendlich ließe sich mit der Forschungsfreiheit des Art. 5 Abs. 3 GG argumentieren. Wenn die geltende Regelung widersprüchlich und der Wortlaut von $\mathbb{s} 291 \mathrm{a}$ SGB V nicht vollständig eindeutig ist, besteht die Pflicht zu verfassungskonformer Auslegung. ${ }^{38}$ Diese könnte unter Heranziehung von Art. 5 Abs. 3 GG zu dem Ergebnis

38 S. dazu allgemein Zippelius 2012, 33, 44; Rüthers/Fischer/Birk 2013, Rn. 763ff.; Lüdemann, JuS 2004, 27ff. m.w.N. (s. zur dogmatischen Begründung ebd., 29). 
führen, dass die Speicherung zusätzlicher Daten zu Forschungszwecken bereits de lege lata ohne die Begrenzung von $\mathbb{2} 291 \mathrm{a}$ Abs. 8 SCB V zulässig ist. ${ }^{39}$

Im Ergebnis ist die vorgenannte Argumentation zwar vertretbar, die weitaus besseren Argumente sprechen aber dafür, dass die Speicherung zusätzlicher Daten zu Forschungszwecken auf der elektronischen Gesundheitskarte nach geltendem Recht ausgeschlossen ist. Hierfür lässt sich bereits der Wortlaut anführen: Die enge Zweckbegrenzung in $\$ 291$ Abs. 1 Satz 3 SGB V verweist zwar auf $\mathbb{2} 291$ S SGB V, dient aber erkennbar nach wie vor dazu, eine Verwendung jenseits der Versorgung soweit wie möglich zu begrenzen. $\mathbb{\$} 291 \mathrm{a}$ Abs. 3 Satz 1 SGB V lässt zwar die Einrichtung weiterer Funktionalitäten der elektronischen Gesundheitskarte zu, $\mathbb{\$} 291 \mathrm{a}$ Abs. 8 SGB V bezieht sich aber ausdrücklich auf die „Daten nach Absatz 2 Satz 1 Nr. 1 oder Absatz 3 Satz 1 “ und schließt damit derartige Zusatzfunktionalitäten mit ein. Ihre Nutzung für andere Zwecke als die der Versorgung der Versicherten ist damit unzulässig. Dies mag im Widerspruch zu der ursprünglichen Absicht des Gesetzgebers stehen, im Rahmen von $\$ 2$ 291a Abs. 3 Satz 1 Nr. 5 SGB V Daten aufzunehmen, die nicht (unmittelbar) der Versorgung dienen. Indes hat diese Absicht im Wortlaut des Gesetzes keinen Niederschlag gefunden. Im Rahmen der Auslegung der Norm kann sie deshalb angesichts des klaren Wortlauts von $\mathbb{2} 291$ a Abs. 8 SGB V nicht maßgeblich sein. Der - bestehende - Widerspruch kann nicht zugunsten der gesetzgeberischen Intention gelöst werden; vielmehr ist eine entsprechende Gesetzesänderung für die Nutzung zu Forschungszwecken zu erwägen. ${ }^{40}$ Dass der Gesetzgeber das Problem zwischenzeitlich erkannt hat, zeigt die im Jahre 2012 eingeführte spezifische Regelung der zusätzlichen Speicherung von Informationen über Organ- und Gewebespenden sowie Vorsorgevollmachten und Patientenverfügungen ( $\$ 291 \mathrm{~A}$ Abs. 3 Satz 1 Nr. 7 bis Nr. 9 SGB V), für die in $₫$ 291a Abs. 5a SCB V detaillierte Bestimmungen zu den einzelnen Zugriffsberechtigungen getroffen wurden.

Auch das Argument der verfassungskonformen Auslegung greift letztlich nicht durch, weil auch gegen die Nutzung zu Forschungszwecken ein Verfassungsprinzip streitet. Die Verwendungsregeln in $\$ 291$ SGB V dienen dem Schutz des Grundrechts auf informationelle Selbstbestimmung der Karteninhaber. Die oben angeführte Auslegung würde letztlich die Beschränkung der Einwilligung durch den Karteninhaber in $\mathbb{} 291 \mathrm{a}$ Abs. 8 SGB V aushebeln. Sie würde es nämlich zulassen, parallel zu den Funktionen in $\$ 291 \mathrm{a}$ Abs. 2 und Abs. 3 SGB V weitere Funktionen zu installieren und mittels einer schlichten Einwilligung medizinische Daten, die an sich - auch - unter Abs. 2 und Abs. 3 fallen, nochmals parallel zu speichern, ohne an $\mathbb{2} 291 \mathrm{a}$ Abs. 8 SGB V gebunden zu sein. Das würde die strikte Zweckbindung letztlich ad absurdum führen. Die Norm ist erkennbar so beabsichtigt und konstruiert, dass sie die Verwendung der auf oder mittels der elektronischen Gesundheitskarte gespeicherten Daten zu anderen Zwecken als denen der Versorgung ausschließen soll, soweit nicht der Gesetzgeber wie bei der Organ- und Gewebespende selbst abweichende Regelungen trifft. Das gilt auch und gerade vor dem Hintergrund der in der öffentlichen Diskussion geäußerten Bedenken hinsichtlich der datenschutzrechtlichen Risiken der elektronischen Gesundheitskarte, denen durch die Norm Rechnung getragen werden sollte.

39 Zur Bedeutung der Forschungsfreiheit s. insoweit BVerfGE 35, 79 (114); 47, 327 (379).

40 S.u. Kap. II.2.3 und bereits Roßnagel/Hornung, MedR 2008, 541ff. 
II Die Nutzung der elektronischen Gesundheitskarte und der Krankenversichertennummer im Forschungskontext

Überdies begrenzen der verfassungsrechtliche Gesetzesvorbehalt und der Bestimmtheitsgrundsatz den Einsatz beliebiger weiterer Funktionen. Die elektronische Gesundheitskarte dient der Verwendung einer Vielzahl sensibler Daten und hat insofern eine erhebliche Eingriffsintensität hinsichtlich des Eingriffs in das Grundrecht auf informationelle Selbstbestimmung. Nach der vom Bundesverfassungsgericht entwickelten Wesentlichkeitslehre muss der Gesetzgeber „in grundlegenden normativen Bereichen, zumal im Bereich der Grundrechtsausübung, soweit diese staatlicher Regelung zugänglich ist, alle wesentlichen Entscheidungen selbst [...] treffen". ${ }^{41}$ Dies gilt zwar direkt nur bei Grundrechtseingriffen durch den Staat. Bei der elektronischen Gesundheitskarte und den mit ihr interagierenden Systemen handelt es sich jedoch um eine so grundsätzliche Infrastruktur, dass aufgrund der besonderen Gefahren für die informationelle Selbstbestimmung der Gesetzgeber seiner verfassungsrechtlichen Schutzpflicht nachkommen und wesentliche Verarbeitungsprozesse auch dann regeln muss, wenn sie durch Private vorgenommen werden. ${ }^{42}$ Die Verwendung der Karte über $\$ 291 a \mathrm{Abs} .2$ und 3 SGB V hinaus - insbesondere wenn diese medizinische Daten umfasst und insofern mit den dort genannten Funktionen zusammenhängt oder sogar interagiert - wird deshalb regelmäßig so wesentlich sein, dass sie gesetzlich geregelt werden muss. ${ }^{43}$ Folglich könnte man höchstens solche Zusatzfunktionen der elektronischen Gesundheitskarte aus dem Anwendungsbereich von $\mathbb{2} 291 \mathrm{~A}$ Abs. 8 SGB V ausnehmen, die keinerlei Gesundheitsdaten speichern. Dies ist bei den hier relevanten Verfahren zur medizinischen Forschung jedoch der Fall.

Hinsichtlich der oben genannten Abgrenzungsprobleme gilt, dass derartige Schwierigkeiten bei der Gesetzesanwendung immer auftreten können. Das hindert den Gesetzgeber aber nicht daran, die neuartigen Risiken der Verwendung von Gesundheitsdaten gerade mittels der elektronischen Gesundheitskarte als besonders gravierend zu werten und diese Wertung in eine - notwendig typisierende - Verwendungsbeschränkung umzusetzen.

Im Ergebnis ist damit eine Speicherung von Forschungsdaten auch als zusätzliche Funktion der elektronischen Gesundheitskarte nach geltendem Recht unzulässig. Die oben zuerst genannte Auslegung überzeugt bereits juristisch nicht. Angesichts der Straf- und Bußgeldandrohungen einer Verletzung von $\$ 291 a$ Abs. 8 SGB V und der zu erwartenden Akzeptanzschwierigkeiten, die die elektronische Gesundheitskarte auch ohne einen Einsatz in der Forschung zu erwarten hat, kann aber auch aus pragmatischen Gesichtspunkten nur davon abgeraten werden, ihr in der Praxis zu folgen.

41 BVerfGE 49, 89 (126); 61, 260 (275); 88, 103 (116); s. Schnapp, in: v. Münch/Kunig, Art. 20 Rn. 76f. m.w.N.; Sachs, in: Sachs, Art. 20 Rn. 116f. m.w.N.

42 Sehr ähnliche Erwägungen stehen letztlich hinter der Entscheidung des BSG, BSGE 102, 134, aus denen das Gericht den Ausschluss der Einwilligung für krankenversicherungsrechtliche Sozialdaten ableitet - s. hierzu oben Kap. II.2.1 sowie näher Roßnagel/Hornung/landt 2009, Teil I, Kap. 1.1.

43 S. Hornung 2005, $212 f$. 
2 Die Nutzung der auf oder mittels der elektronischen Gesundheitskarte gespeicherten medizinischen Daten

\title{
2.2.3 Unterscheidung zwischen Ärzten und Nicht-Ärzten?
}

\author{
Muss bei Forschern zwischen Ärzten und Nicht-Ärzten bezüglich des Zugriffs auf \\ Behandlungs- oder Forschungsdaten zum Zwecke der Forschung unterschieden \\ werden?
}

Im Kontext der Nutzung der elektronischen Gesundheitskarte zu Forschungszwecken ist eine weitere relevante Frage, ob bezüglich des Zugriffs auf Behandlungs- oder Forschungsdaten zum Zwecke der Forschung zwischen Ärzten und Nicht-Ärzten unterschieden werden muss.

Diese Frage muss nach geltendem Recht verneint werden, da der Zugriff auf Behandlungsdaten zu Forschungszwecken sowie die Speicherung von und der Zugriff auf Forschungsdaten auf oder mittels der elektronischen Gesundheitskarte derzeit unzulässig sind (s.o. Kap. II.2.2.1 und II.2.2.2). Das gilt sowohl für Forscher, die zugleich Ärzte sind, als auch für sonstige Forscher.

Zum besseren Verständnis seien hier dennoch die derzeit geltenden Zugriffsbefugnisse genannt:

- Der Zugriff auf die Behandlungsinformationen nach $\mathbb{2} 291 \mathrm{a}$ Abs. 3 Satz 1 Nr. 1 bis Nr. 5 SGB V steht gemäß $\$ 291 a$ Abs. 4 Satz 1 Nr. 2 SGB V ausschließlich dem oben in Kapitel II.2.2.2.1 genannten Personenkreis - also nicht nur Ärzten, sondern auch einer Reihe sonstiger Personen aus dem medizinischen Umfeld - offen. Im Fall der Informationen über Organ- und Gewebespenden sowie Vorsorgevollmachten und Patientenverfügungen ( $\$ 291$ a Abs. 3 Satz 1 Nr. 7 bis Nr. 9 SGB V) trifft $\mathbb{2} 291 \mathrm{a}$ Abs. 5a SGB V eine noch engere Regelung (Beschränkung auf Ärzte und deren berufsmäßige Gehilfen). Anderen Personen ist der Zugriff untersagt; ein Verstoß stellt gemäß $\$ 307 \mathrm{~b}$ SGB V eine Straftat dar.

- Hinsichtlich des Zugriffs auf Funktionen, die über $\$ 291 \mathrm{a}$ Abs. 2 und 3 SGB V hinausgehen, stellt sich im Rahmen der Anwendung von $\$ 291 \mathrm{a}$ Abs. 8 SGB V hinsichtlich der zugriffsberechtigten Personen das parallele Problem zur oben behandelten Frage der zugelassenen Zwecke des Zugriffs. ${ }^{44}$ Die Frage muss hier identisch beantwortet werden: Es ließe sich zwar vertreten, $\mathbb{s} 291 \mathrm{~A}$ Abs. 8 SGB V erfasse nur den Zugriff auf die Funktionen nach $\$ 291 \mathrm{a}$ Abs. 2 und Abs. 3 SGB V. Dies würde jedoch für medizinische Daten eine Umgehung der Norm eröffnen, die der Gesetzgeber erkennbar nicht zulassen wollte. Auch der Zugriff auf zusätzliche Funktionen ist deshalb nach geltendem Recht - jedenfalls soweit Daten verwendet werden, die auch unter die Anwendungen nach $\mathbb{2} 291 \mathrm{a}$ Abs. 2 und 3 SGB V fallen könnten - nur dem in $\$ 291$ a Abs. 4 Satz 1 Nr. 2 beziehungsweise Abs. 5a SGB V genannten Personenkreis offen.

44 S. Kap. II.2.2.2.2 
II Die Nutzung der elektronischen Gesundheitskarte und der Krankenversichertennummer im Forschungskontext

\subsubsection{Gesamtergebnis de lege lata}

Als Gesamtergebnis lässt sich festhalten, dass eine Verwendung der elektronischen Gesundheitskarte zu Forschungszwecken nach geltendem Recht unzulässig ist. Dies gilt auch im Falle einer Einwilligung des Karteninhabers

\section{3 Änderung von § 291a Abs. 8 SGB V?}

Der Gesetzgeber hat die Regelungen zur elektronischen Gesundheitskarte in hoher Frequenz geändert und dadurch zu erkennen gegeben, dass er bereit ist, auf Einführungsprobleme und sinnvolle Erweiterungen der Funktionalitäten durch eine entsprechende gesetzliche Ausgestaltung zu reagieren. ${ }^{45}$ Dementsprechend stellt sich die Frage der rechtspolitischen Bewertung der Situation de lege lata.

\subsubsection{Hintergrund}

Das in Kapitel II.2.2 gefundene Ergebnis erscheint aus mehrfacher Sicht unbefriedigend. Zunächst verhindert es medizinische Forschung - die sowohl im Interesse der einzelnen aktuellen und künftigen Patienten, als auch im gesamtgesellschaftlichen Interesse eines funktionsfähigen Gesundheitswesens ist - auf der Basis der Telematik-Infrastruktur, die hierfür besonders geeignet wäre.

Darüber hinaus wiesen die Regelungen bis zum Jahre 2012 hinsichtlich der zu untersuchenden Frage Inkonsistenzen auf. ${ }^{46} \$ 291 \mathrm{a}$ Abs. 8 SCB V beschränkt die Verwendung der auf oder mittels der elektronischen Gesundheitskarte gespeicherten Daten auf die Zwecke der Versorgung des Patienten (einschließlich der Abrechnung der zum Zwecke der Versorgung erbrachten Leistungen). Die Gesetzesbegründung nennt aber als Anwendungsfälle für $\$ 291 a$ Abs. 3 Satz 1 Nr. 5 SCB V Daten, deren Verwendung über die Versorgung hinausgeht (Patientenverfügungen und Organspendeausweis). ${ }^{47}$ Der Gesetzgeber hat diese Inkonsistenz zum Anlass genommen, den Bereich in $\$ 291 \mathrm{a}$ Abs. 3 Satz 1 Nr. 7 bis 9 und Abs. 5a SGB V nunmehr spezialgesetzlich zu regeln; dies könnte für gleichfalls sinnvolle andere Funktionalitäten ebenso erfolgen.

Schließlich können Versicherte immer noch in die Verwendung von Behandlungsdaten zu Forschungszwecken einwilligen, die zuvor zu Behandlungszwecken auf oder mittels der elektronischen Gesundheitskarte transportiert wurden, vorausgesetzt, die Daten wurden nicht im Rahmen eines Umgehungstatbestands und nur zum Zwecke der Weiterleitung von der elektronischen Cesundheitskarte in ein anderes, zulässiges System kopiert. ${ }^{48}$ Auch die Unsicherheiten, die diese Abgrenzung mit sich bringt, sprechen für eine Veränderung der derzeitigen Rechtslage.

Die folgenden Überlegungen haben keinen abschließenden Charakter. In einem so sensiblen Bereich wie der Forschung mit Gesundheitsdaten von Patienten ist vor

45 \291a SGB V gilt derzeit in der 14. (!) Fassung, obwohl die elektronische Gesundheitskarte in der Praxis der Versorgung bisher keinerlei Rolle spielt.

46 S.0. Kap. II.2.2.2.2.

47 S. Fn. 22.

48 S. Kap. II.2.2.2.2. 
2 Die Nutzung der auf oder mittels der elektronischen Gesundheitskarte gespeicherten medizinischen Daten

einem Handeln des Gesetzgebers eine möglichst breite gesellschaftliche und politische Diskussion unabdingbar. Hinzu kommt, dass die elektronische Gesundheitskarte bereits ohne die Nutzung zu Forschungszwecken sowohl in der Ärzteschaft wie unter den Versicherten umstritten und der Einsatz in der Forschung bislang - soweit ersichtlich - nicht diskutiert worden ist. ${ }^{49}$ Dementsprechend verstehen sich die folgenden Ausführungen als Anstoß zu einer rechtspolitischen Diskussion.

\subsubsection{Rechtspolitische Ausgangslage}

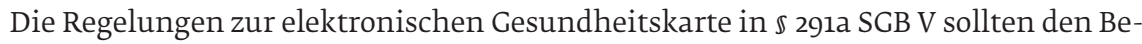
teiligten im Gesundheitswesen frühzeitig Planungssicherheit für die Einführung der Karte und den Aufbau der mit ihr interagierenden Infrastruktur geben. Dem Gesetzgeber war dabei aber bewusst, dass sich im Laufe der Erforschung und Implementierung die Notwendigkeit neuer, ergänzender und veränderter Regelungen ergeben würde. In der Begründung zu $₫$ 291a SGB V heißt es:

„Zur umfassenden Nutzung der elektronischen Gesundheitskarte sind insbesondere unter Berücksichtigung von Erfahrungen aus der Testphase weitergehende rechtliche Regelungen zu treffen." ${ }^{50}$

Im Folgenden ist zu untersuchen, inwieweit eine Veränderung der strikten Regelung in $\mathbb{2} 291$ a Abs. 8 SGB V zum einen rechtspolitisch wünschenswert, zum anderen verfassungsrechtlich zulässig wäre.

\subsubsection{Grundsätzliche rechtspolitische Bewertung einer Änderung}

Medizinische Forschung ist zwar in weiten Teilen ohne personenbezogene Daten möglich. ${ }^{51}$ In vielen Konstellationen bedarf es aber zumindest der Verwendung von Pseudonymen, um eine kontinuierliche Zusammenführung von Behandlungsinformationen einer Person zu gewährleisten..$^{52}$ Überdies wird in vielen Fällen ein Personenbezug entweder direkt bestehen (etwa bei der Forschung durch einen behandelnden Arzt) oder herstellbar sein (bei kleinen Patientengruppen oder ungewöhnlichen Krankheiten).

Bedeutung und Wert wissenschaftlicher Forschung kommen in der Crundrechtsgarantie des Art. 5 Abs. 3 GG und internationalen und europäischen Menschenrechtsgarantien zum Ausdruck. ${ }^{53}$ Das Bundesverfassungsgericht hat in mehreren Entscheidungen den Wert von Wissenschaft und Forschung „sowohl für die Selbstverwirklichung des Einzelnen als auch für die gesamtgesellschaftliche Entwicklung " 54 hervorgehoben und betont, „dass gerade eine von gesellschaftlichen Nützlichkeits- und politischen Zweckmäßigkeitsvorstellungen befreite Wissenschaft dem Staat und der Gesellschaft im Ergebnis am besten dient". ${ }^{55}$ Die gesellschaftliche Bedeutung von

\footnotetext{
49 S. für Auszüge aus dem Folgenden Roßnagel/Hornung, MedR 2008, $541 \mathrm{ff}$.

50 BT-Drs. $15 / 1525,144$

51 Bäumler, MedR 1998, 400, 405; s. bereits Beier 1979, 107; Lilie 1980, 103f.; s.a. Roßnagel/Hornung/Jandt 2009 , Teil I, Kap. 2.

52 S. z.B. Gerling, in: Roßnagel, Handbuch Datenschutzrecht, Kap. 7.10, Rn. $26 f$.

53 S. zu letzteren Bethge, in: Sachs, Grundgesetz, Art. 5 Rn. $6 \mathrm{ff}$.

54 BVerfGE 35, 79 (114).

55 BVerfGE 47, 327 (379).
} 
II Die Nutzung der elektronischen Gesundheitskarte und der Krankenversichertennummer im Forschungskontext

Wissenschaft und Forschung wird gerade im medizinischen Bereich besonders deutlich.

Angesichts dieser Dimension und der oben in Kapitel II.2.3.1 dargelegten Inkonsistenzen und Lücken des geltenden Rechts, die für die auf oder mittels der elektronischen Gesundheitskarte zu Behandlungszwecken übermittelten Daten letztlich doch eine Nutzung zu Forschungszwecken zulassen, erscheint eine explizite gesetzliche Regelung rechtspolitisch wünschenswert.

Gleichzeitig ist deutlich, dass jede Erweiterung der nach $₫$ 291a SGB V derzeit zugelassen Verwendungszwecke der elektronischen Gesundheitskarte mit äußerster Vorsicht erfolgen sollte. Bei allen Vorteilen, die die elektronische Gesundheitskarte perspektivisch bietet, ${ }^{56}$ bestehen vergleichbar große Risiken für die informationelle Selbstbestimmung der Karteninhaber. Auch diese hat eine gesellschaftliche Dimension: Das Bundesverfassungsgericht hat betont, dass das Vertrauensverhältnis zwischen Versichertem und Leistungserbringer „Grundvoraussetzung ärztlichen Wirkens [ist], das die Chancen der Heilung vergrößert und insgesamt der Aufrechterhaltung einer leistungsfähigen Gesundheitsfürsorge dient “. ${ }^{77}$ Ebenso hat der Europäische Gerichtshof für Menschenrechte darauf hingewiesen, dass ein beschädigtes Vertrauen in die Verschwiegenheit der Leistungserbringer Gefahren für die gesamte Bevölkerung hervorrufen kann. ${ }^{58}$

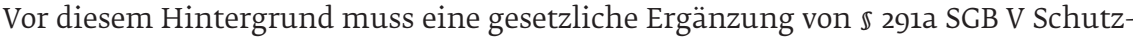
mechanismen zur Wahrung von Patientenautonomie und informationeller Selbstbestimmung der Karteninhaber enthalten und darf die technischen Schutzvorkehrungen, die die Norm vorsieht, nicht aushebeln. Dass dem Gesetzgeber diese Voraussetzungen bewusst sind, zeigt die schon erwähnte Erweiterung der Funktionalitäten in $\mathbb{2} 291$ a Abs. 3 Satz 1 Nr. 7 bis 9 SGB V, für die in $\mathbb{2} 291$ a Abs. 5a SGB V spezifische Zugriffsregelungen normiert wurden.

\subsubsection{Inhalt einer gesetzlichen Ergänzung von \ 291a SGB V}

\subsubsection{Verhältnis zu § 291a Abs. 8 SGB V}

Eine „Forschungsklausel“ im Rahmen von $\$ 291 a$ SCB V müsste zunächst das Verhältnis zu $\mathbb{2} 291 \mathrm{a}$ Abs. 8 SGB V klären. Dies könnte dadurch erfolgen, dass in diesem im zweiten Halbsatz des ersten Satzes („mit ihnen darf nicht vereinbart werden, Derartiges zu gestatten“) hinter „darf“ die Formulierung „,- vorbehaltlich Absatz 9 -“ eingefügt würde. Eine Veränderung des ersten Halbsatzes ist nicht erforderlich, da eine Einwilligung vom Karteninhaber nicht „,verlangt“ wird. Das absolute Verbot in

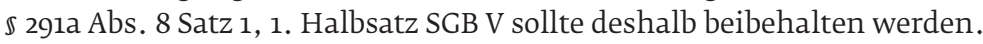

56 Etwa für eine verbesserte Versorgung durch eine höhere Verfügbarkeit von Daten für den behandelnden Arzt (etwa zum Erkennen von Kontraindikationen), eine verbesserte Arzneimittelsicherheit und eine geringere Eingriffsintensität, wenn zum Beispiel auf erneute Untersuchungen verzichtet werden kann oder erkannt wird, dass eine bestimmte Behandlung überflüssig ist; s. Hornung 2005, 44f. m.w.N.

57 BVerfGE 32, 373 (380); s.a. Ulsenheimer/Heinemann, MedR 1999, 197, 202; Lilie 1980, 78f.; Beier 1979, 55; Schirmer, in: Roßnagel, Handbuch Datenschutzrecht, Kap. 7.12, Rn. 23; zu diesem Gedanken bereits Schmidt, NJW 1962, 1745, 1747; Laufs, NJW 1975, 1433, 1434; s.a. Roßnagel, NJW 1989, 2303, 2306; Hornung 2005, 229.

58 Z ./. Finnland, Urteil v. 25.2.1997 (abrufbar unter http://www.echr.coe.int/Eng/Judgments.htm), Abs. 95. 


\subsubsection{Zweckbestimmung für den Zugriff}

Der Grundsatz der datenschutzrechtlichen Zweckbindung fußt im Verhältnismäßigkeitsprinzip. Er ist deshalb im Bereich hoheitlicher Datenverwendungen ein Verfassungsgebot; Zweckentfremdungen sind verfassungsrechtlich unzulässig. ${ }^{59} \mathrm{Da}$ eine Datenverwendung immer nur in Bezug auf einen konkreten Zweck hin geeignet sein kann, muss dieser zuvor eindeutig und ausdrücklich bestimmt werden. ${ }^{60}$ In der Folge dürfen die Daten nur zu diesem Zweck verarbeitet und genutzt werden. Nachträgliche Zweckänderungen, die zu einer Datenverwendung außerhalb des ursprünglichen Zwecks führen, stellen einen selbständigen Grundrechtseingriff dar. ${ }^{61}$ Dieser neue Eingriff unterliegt den üblichen Anforderungen. Es ist also eine gesetzliche Ermächtigungsgrundlage oder eine Einwilligung erforderlich. Damit wird verhindert, dass Daten zu einem eng begrenzten Zweck erhoben und danach zu anderen, unbestimmten Zwecken verwendet werden. ${ }^{62}$

Der hier relevante Zweck der medizinischen Forschung sollte als solcher bezeichnet und im Gesetz verankert werden. In Anlehnung an die Formulierung in $\mathbb{} 40$ BDSC („wissenschaftliche Forschung“) - die verhindern soll, dass vorgeblich forschende, in Wahrheit aber kommerzielle Tätigkeiten vorliegen - könnte man den Zweck auf die „wissenschaftlich-medizinische Forschung“ begrenzen.

\subsubsection{Einwilligungserfordernis}

Die Verwendung personenbezogener Daten bedarf gemäß $\$ 4$ Abs. 1 BDSC einer gesetzlichen Ermächtigung oder Einwilligung. Angesichts der Sensibilität der auf oder mittels der elektronischen Gesundheitskarte gespeicherten Daten wäre es verfassungsrechtlich kaum haltbar (und unter Akzeptanzgesichtspunkten ${ }^{63}$ potentiell desaströs), ihre Verwendung ohne die ausdrückliche Einwilligung der Karteninhaber zuzulassen, denn die Einwilligung ist Ausdruck des Rechts auf informationelle Selbstbestimmung der betroffenen Person. Dementsprechend ist ein Einwilligungserfordernis ausdrücklich vorzusehen. Gemäß allgemeinen Regeln muss die Einwilligung informiert und freiwillig abgegeben werden; die Anforderungen entsprechen insoweit den allgemeinen Bedingungen für Einwilligungserklärungen zur Verwendung von Daten im Kontext medizinischer Forschung. ${ }^{64}$ Dabei sind unter Umständen Sondervorschriften in einigen fachspezifischen Gesetzen zu beachten. ${ }^{65}$

Die Einwilligung ist schriftlich zu erteilen. Das gilt bereits nach geltendem Recht für alle freiwilligen Anwendungen der elektronischen Cesundheitskarte. Ein Schriftformerfordernis ergibt sich zwar nicht direkt aus $\mathbb{s} 291 \mathrm{a}$ SCB V, wohl aber aus $\mathbb{4} 4 \mathrm{a}$ Abs. 1 Satz 3 BDSG, der subsidiär anwendbar ist. Jede andere Form der Einwilligung wäre angesichts der Komplexität der Funktionsweise der elektronischen Gesundheits-

\footnotetext{
59 S. Di Fabio, in: Maunz/Dürig, Grundgesetz, Art. 2 Abs. 1 Rn. 186; Podlech, in: Denninger et al., Alternativkommentar Grundgesetz, Art. 2 Abs. 1 Rn. 82; Bizer 1992, 148; v. Zezschwitz, in: Roßnagel, Handbuch Datenschutzrecht, Kap. 3.1, Rn. 3; kritisch gegenüber der Effektivität des Zweckbindungsgrundsatzes Trute, in: Roßnagel, Handbuch Datenschutzrecht, Kap. 2.5, Rn. $37 f f$.

60 BVerfGE 65, 1, (46); Gola/Schomerus 2012, \&14 Rn. 9.

61 Roßnagel/Pfitzmann/Garstka 2001, 115; Scholz 2003, 139 m.w.N.

62 Denninger, KJ 1985, 215, 220.

63 S. zu den Akzeptanzfragen der elektronischen Gesundheitskarte Hornung 2005, 421ff.

64 S. dazu Roßnagel/Hornung/Jandt 2009, $3 f f$.

65 S. näher Roßnagel/Hornung/Jandt 2009, $17 f$.
} 
II Die Nutzung der elektronischen Gesundheitskarte und der Krankenversichertennummer im Forschungskontext

karte nicht angemessen. ${ }^{66}$ Das gilt erst recht für die Verwendung zu Forschungszwecken. Die Aufklärung der Karteninhaber wäre nach einer etwaigen Gesetzesänderung bereits nach $\$ 291 \mathrm{a}$ Abs. 3 Satz 3 SGB V erforderlich, der eine Unterrichtung über (alle) Funktionalitäten der elektronischen Gesundheitskarte verlangt.

Analog zu der Einwilligung in die Verwendung der elektronischen Gesundheitskarte zu den freiwilligen Anwendungen ( $\$ 291 \mathrm{a}$ Abs. 3 Satz 5, 1. Halbsatz SGB V) sollte die Einwilligung der Nutzung zu Forschungszwecken auf der elektronischen Gesundheitskarte selbst dokumentiert werden. Dies muss für jedes Forschungsvorhaben spezifisch erfolgen.

\subsubsection{Zugriffsberechtigte}

Nach geltendem Recht ist der Zugriff auf die Karte gemäß $\$ 291 a$ Abs. 4 SGB V Personen vorbehalten, die im Rahmen der medizinischen Versorgung tätig sind (vor allem Ärzte, Zahnärzte, Apotheker, Psychotherapeuten, sonstige Angehörige eines Heilberufs und eine Reihe von Hilfspersonen); ${ }^{67}$ für die Erklärungen zur Organ- und Gewebespende sowie zu Vorsorgevollmachten und Patientenverfügungen ist der Kreis der Zugriffsberechtigten in $\mathbb{2} 291$ a Abs. 5a SGB V weiter eingeengt. Damit stellt sich die Frage, ob im Rahmen des Zugriffs zu Forschungszwecken eine identische Beschränkung gelten soll, ob weitere Personen berechtigt sein könnten oder ob der berechtigte Personenkreis sogar in das Belieben des Karteninhabers gestellt werden sollte.

Gegen eine Beschränkung auf Personen, die im Bereich der Versorgung tätig sind, spricht eine mögliche Behinderung der Forschungsarbeit. Außerdem könnte es zu Ungleichbehandlungen kommen, wenn bestimmte Forscher oder Forschungsinstitutionen zu dem genannten Personenkreis zählen, andere jedoch nicht. Auf der anderen Seite hat die Beschränkung auf den in $\mathbb{2} 291 \mathrm{a}$ Abs. 4 SGB V genannten Personenkreis den Vorteil der Eindeutigkeit. Angesichts der erheblichen Grundrechtsrelevanz der elektronischen Gesundheitskarte und der Sensibilität der gespeicherten Daten ist unbedingt zu verhindern, dass unberechtigte Dritte Zugriff auf die Daten erhalten. Darüber hinaus ist die sichere und effiziente Versorgung des Karteninhabers Hauptzweck der elektronischen Gesundheitskarte. Das abgestufte Zugriffssystem in $\$ 291 \mathrm{a}$ Abs. 4 SGB V sollte nicht ohne Not aufgrund sekundärer Einsatzfelder der elektronischen Gesundheitskarte aufgegeben oder abgeschwächt werden.

Hierfür spricht letztlich auch entscheidend der technische Schutz der Karte. Gemäß $\$ 291 a$ Abs. 5 Satz 3 SGB V wird der Zugriff auf die Daten des elektronischen Rezepts und der freiwilligen Anwendungen nach $\$ 291 \mathrm{a}$ Abs. 3 SGB V nur in Verbindung mit einem elektronischen Heilberufsausweis, im Falle des elektronischen Rezepts auch in Verbindung mit einem entsprechenden Berufsausweis erfolgen, die zur elektronischen Authentifizierung und zur Erstellung qualifizierter elektronischer Signaturen geeignet sind. ${ }^{68}$ Dasselbe gilt nach $\mathbb{} 291 a$ Abs. 5a Satz 1 SGB V für Erklärungen zu

66 S. für die bisherigen Anwendungen der elektronischen Gesundheitskarte Weichert, DuD 2004, 391, 399; Hornung 2005, 62; a.A. Schneider 2004, 153.

67 S. näher Hornung 2005, 220ff.; Bales/Dierks/Holland/Müller 2007, $96 \mathrm{ff}$.

68 Für berufsmäßige Gehilfen gilt $\$ 291$ a Abs. 5 Satz 4 BDSG. Danach ist ein Zugriff auch ohne elektronischen Berufsausweis zulässig, wenn eine Autorisierung durch einen Berufsausweisträger vorliegt und nachprüfbar elektronisch protokolliert wird, wer auf die Daten zugegriffen hat und von welcher Person die zugreifende Person autorisiert wurde. 
Organ- und Gewebespende sowie zu Vorsorgevollmachten und Patientenverfügungen. ${ }^{69}$ Nur auf die Daten des „Patientenfachs“ nach $\$ 291 a$ Abs. 3 Satz 1 Nr. 5 SGB V können die Versicherten auch mittels einer eigenen qualifizierten Signaturkarte zugreifen; für die regelmäßig nicht-gesundheitsbezogenen Informationen zu Organund Gewebespende sowie zu Vorsorgevollmachten und Patientenverfügungen genügt gemäß $\$ 291 a$ Abs. 5a Satz 4 SCB V auch ein sonstiges geeignetes Authentifizierungsverfahren. Wenn nun auch Personen aus dem Forschungsumfeld auf die Daten zugreifen sollen, die nicht unter $\mathbb{S} 291 \mathrm{a}$ Abs. 4 oder Abs. 5a SGB V fallen, so müssten diese entweder mit entsprechenden Berufsausweisen oder sonstigen Chipkarten ausgestattet werden, mit denen sie sich gegenüber der elektronischen Gesundheitskarte authentisieren, oder die Sicherheitsmechanismen der Karte müssten abgesenkt werden.

Die zweite Lösung erscheint unter Datenschutz- und Datensicherheitsgesichtspunkten verfassungsrechtlich nicht akzeptabel. Der elektronische Umgang mit den Patientendaten mittels einer Telematik-Infrastruktur greift in erheblichem Umfang in die Grundrechte der Patienten ein und genügt nur unter den Bedingungen rechtlicher und technischer Sicherungsmittel verfassungsrechtlichen Anforderungen. Aber auch die Vergabe von Zugriffsbefugnissen über den in $\$ 291$ a Abs. 4 und Abs. 5a SCB V genannten Personenkreis hinaus ist nicht ratsam. Sie würde die Vergabe der Chipkarten, die zum Zugriff berechtigen, erschweren und unübersichtlich machen. Diese Aufgabe wird gemäß $\mathbb{2} 291 \mathrm{a}$ Abs. 5c SGB V von den Ländern auf bestimmte Stellen - vor allem die Berufskammern ${ }^{70}$ - übertragen, die hierfür besonders geeignet sind. Außerdem würde der Kreis der Personen, die faktisch auf elektronische Gesundheitskarten zugreifen können, erheblich erweitert und für die Karteninhaber intransparent gemacht. Die verbleibenden Einschränkungen für Forschungsinstitute, die nicht über Heilberufsausweisträger verfügen, müssen - auch angesichts des Hauptzwecks der elektronischen Gesundheitskarte als Instrument zur Verbesserung der Versorgung hingenommen werden. Dies erscheint vor allem deshalb akzeptabel, weil diese Fälle in der Praxis selten sein dürften.

\subsubsection{Technische Schutzmechanismen beim Zugriff}

Wie bereits im vorherigen Abschnitt ausgeführt, sollten die technischen Schutzmechanismen der elektronischen Gesundheitskarte nicht wegen einer Verwendung zu Zwecken medizinischer Forschung abgeschwächt werden. Das gilt sowohl für die Person des Zugreifenden (der über einen gültigen Heilberufsausweis verfügen muss), als auch für die Person des Inhabers der elektronischen Gesundheitskarte. Dieser muss nach geltendem Recht beim Zugriff mitwirken. $\mathbb{\$} 291 \mathrm{a}$ Abs. 5 Satz 2 SGB V bestimmt: „Durch technische Vorkehrungen ist zu gewährleisten, dass in den Fällen des Absatzes 3 Satz 1 Nr. 2 bis 6 der Zugriff nur durch Autorisierung der Versicherten möglich ist“. Lediglich beim elektronischen Rezept nach $\mathbb{2}$ 291a Abs. 2 Satz 1 Nr. 1 SGB V und den Notfallinformationen nach $\$ 291$ a Abs. 3 Satz 1 Nr. 1 SGB V besteht der Schutz nur im Besitz der elektronischen Gesundheitskarte; hinzu treten seit dem

69 Für diese gilt nach $\S 291 \mathrm{a}$ Abs. 5a Satz 1, 2. Halbsatz SGB V ebenfalls die Ausnahme hinsichtlich der berufsmäßigen Gehilfen (s. Fn. 68).

70 S. näher Bales/Dierks/Holland/Müller 2007, 114ff.; Scholz, in: Rolfs/Giesen/Kreikebohm/Udsching, BeckOK SGB V § 291a, Rn. 16; Hornung, in: Hänlein/Kruse/Schuler, § 291a Rn. 18. 
II Die Nutzung der elektronischen Gesundheitskarte und der Krankenversichertennummer im Forschungskontext

Jahre 2012 die eng begrenzten Fälle der Organ- und Gewebespende (im Falle der Feststellung des Todes) sowie der Vorsorgevollmacht und Patientenverfügung, soweit keine Einwilligungsfähigkeit vorliegt.

Nach dem gegenwärtigen Stand der Technik wird die Autorisierung nach $\$ 291 \mathrm{~A}$ Abs. 5 Satz 2 SCB V mittels einer PIN erfolgen. Hieran sollte auch im Kontext medizinischer Forschung festgehalten werden.

\subsubsection{Schutz der Zweckbindung}

Ein besonderer Schutz der Zweckbindung durch Vorschriften des Straf- und Ordnungswidrigkeitenrechts erscheint nicht erforderlich. Durch die Anbindung einer Forschungsklausel an $\$ 291$ A Abs. 8 SGB V (s. den Vorschlag oben unter Kap. II.2.3.4.1) würden bei einer Nutzung der Daten zu anderen Zwecken als denen der wissenschaftlich-medizinischen Forschung die Schutzvorschriften der $\mathbb{S} 291 \mathrm{a}$ Abs. 8, 307, 307b SGB V eingreifen.

\subsubsection{Speicherung zusätzlicher Forschungsdaten}

Die Frage der Speicherung zusätzlicher Forschungsdaten verliert in dem Moment an Brisanz, in dem die Nutzung der ohnehin gespeicherten Daten unter bestimmten Bedingungen zugelassen wird. Sie bleibt dort relevant, wo Daten für die Forschung verwendet werden, die keinen - oder keinen unmittelbar gesicherten - Zusammenhang mit der Behandlung haben. Wenn dieses Vorgehen zur Forschung unabdingbar ist, sollte es unter denselben Voraussetzungen wie die Nutzung der Behandlungsdaten zugelassen werden.

Dies kann allerdings nur unter einer wichtigen Bedingung gelten: Die Funktionen der elektronischen Gesundheitskarte, die zum Zwecke der Versorgung in $\$ 291$ SGB V durch Gesetz entweder vorgeschrieben oder ermöglicht werden, dürfen nicht beeinträchtigt werden. Das gilt sowohl hinsichtlich der Funktionen, die sich nicht gegenseitig behindern dürfen, als auch hinsichtlich etwaiger Probleme mit der Speicherkapazität. Um dies zu verhindern, ist eine Zulassung nach technischer Prüfung vorzusehen.

\subsubsection{Beschlagnahmeschutz}

Soweit auf der elektronischen Gesundheitskarte gespeicherte Behandlungsinformationen zu Zwecken der medizinischen Forschung genutzt werden, fallen sie weiterhin und im selben Umfang wie bisher unter den Beschlagnahmeschutz gemäß $\mathbb{9} 97$ Abs. 1 Nr. 2, Abs. 2 Satz 1 StPO. ${ }^{71}$ Ein Sonderproblem könnte dagegen auftreten, wenn wie im letzten Abschnitt vorgeschlagen - zusätzliche Forschungsdaten gespeichert werden. $\mathbb{S} 97$ Abs. 1 Nr. 2 StPO gilt nur für Umstände, „auf die sich das Zeugnisverweigerungsrecht erstreckt“. Das verweist auf $\mathbb{5} 53$ Abs. 1 Satz 1 Nr. 3 StPO und beschränkt sich auf das, was Berufsgeheimnisträgern (unter anderem Ärzten) ,, in dieser Eigenschaft anvertraut oder bekannt geworden ist“".

71 S. dazu Hornung 2005, 233ff.; Bales/Dierks/Holland/Müller 2007, 168ff. 
Folglich werden Daten, die ausschließlich zu Forschungszwecken erhoben werden und insofern auch durch Nicht-Ärzte hätten erhoben werden können - zumindest nicht stets unter den Beschlagnahmeschutz fallen. ${ }^{72}$ Das würde nach aktueller Rechtslage auch für die elektronische Gesundheitskarte gelten. Im Grenzbereich zwischen Konsiliarbehandlung und Forschung können überdies Unklarheiten über die Reichweite des Beschlagnahmeschutzes entstehen.

Es könnte deshalb erwogen werden, diesen Schutz ohne die oben genannte Einschränkung direkt an die elektronische Gesundheitskarte selbst zu knüpfen und sämtliche auf ihr gespeicherten Daten von der Beschlagnahme auszuschließen. Auf der anderen Seite erscheint eine solche Ausdehnung - auch unter Berücksichtigung der Vorgaben des Bundesverfassungsgerichts, wonach derartige Vorschriften zumindest tendenziell eng zu fassen sind 73 - nicht zwingend erforderlich. Zu Forschungszwecken erhobene Daten sind auch heute nicht vom Beschlagnahmeschutz erfasst. Es müsste allerdings sichergestellt werden, dass nicht im Zuge von Zugriffen auf diese Daten gleichzeitig Daten aus der elektronischen Gesundheitskarte ausgelesen werden, die einem Arzt in genau dieser Eigenschaft offenbart wurden und insofern unter $₫ 97$ Abs. 1 Nr. 2, Abs. 2 StPO fallen. Wenn dies nicht sichergestellt werden kann, hat der Zugriff insgesamt zu unterbleiben.

Die im vorstehenden Absatz genannten Einschränkungen können allerdings bereits im Wege der Auslegung dem geltenden Recht entnommen werden. Insofern erscheint auch eine Präzisierung der Rechtslage zumindest nicht zwingend erforderlich.

\subsubsection{Vorgeschlagener Wortlaut}

Entsprechend den vorstehenden Ausführungen könnte der Wortlaut für einen Absatz über die medizinische Forschung in $\$ 291$ S SGB V wie folgt lauten:
„(9) Die Nutzung von Daten nach Absatz 2 Satz 1 Nr. 1 und Absatz 3 Satz 1 mittels der elekt- ronischen Gesundheitskarte ist unter den technischen Voraussetzungen von Absatz 5 auch zu Zwecken der wissenschaftlich-medizinischen Forschung zulässig, wenn die Versicherten in diese Verwendung schriftlich einwilligen. Die Speicherung zusätzlicher Forschungsdaten kann unter denselben Voraussetzungen zugelassen werden, wenn sie die sonstigen Funktio- nen der elektronischen Gesundheitskarte nicht behindert. Die Einwilligung ist bei derjeweils ersten Verwendung der Karte zu Forschungszwecken auf der Karte zu dokumentieren und jederzeit widerruflich.“

Es wird nochmals betont, dass die vorstehenden Überlegungen und der vorgeschlagene Wortlaut angesichts der Sensibilität der verwendeten Daten und der Diskussionen, die die elektronische Gesundheitskarte bereits ohne den Einsatz zu Forschungszwecken ausgelöst hat, nicht Ergebnis, sondern Ausgangspunkt einer rechtspolitischen Debatte sein sollten.

72 Das Zeugnisverweigerungsrecht gilt nur für Tatsachen mit unmittelbarem Bezug zur Berufsausübung, s. näher Meyer-Goßner 2014, $\$ 53$ Rn. 7 ff. m.w.N.

73 So muss etwa der Kreis der Zeugnisverweigerungsberechtigten wegen der Notwendigkeit, eine funktionsfähige Rechtspflege zu erhalten, auf das unbedingt erforderliche Maß begrenzt werden, s. BVerfGE 33, 367 (383); 38, 312 (321). 
II Die Nutzung der elektronischen Gesundheitskarte und der Krankenversichertennummer im Forschungskontext

\subsection{Rechtslage nach einer hypothetischen Gesetzesänderung}

Im Folgenden soll kurz dargestellt werden, wie sich die vorgeschlagene Gesetzesänderung auf die Beantwortung der Fragen in den Kapiteln II.2.2.1 bis II.2.2.3 auswirken würde, die oben nach geltendem Recht behandelt wurden.

\subsubsection{Zugriff von Forschern auf Behandlungsdaten mit Einverständnis des Patienten}

Die Frage, ob Forscher mit Einverständnis des Patienten Zugriff auf vorhandene Behandlungsdaten bekommen dürfen (oben Kap. II.2.2.1), wäre de lege ferenda gemäß dem vorstehend vorgeschlagenen Wortlaut eines $\mathbb{2} 291 \mathrm{a}$ Abs. 9 SGB V n.F. wie folgt zu beantworten: Der Zugriff wäre nach einer ausdrücklich schriftlich erklärten und auf der elektronischen Gesundheitskarte dokumentierten Einwilligung des Patienten zulässig. Das würde allerdings nicht für alle Forscher gelten (s.u. Kap. II.2.4.3).

\subsubsection{Speicherung zusätzlicher Daten}

Zusätzliche Forschungsdaten dürften nach dem Vorschlag für Zwecke der Forschung mit Einverständnis des Patienten auf der elektronischen Gesundheitskarte gespeichert und verwaltet werden (s. Frage in Kap. II.2.2.2), wenn dies zugelassen wird und die sonstigen Anwendungen der elektronischen Gesundheitskarte weder funktional noch kapazitätsmäßig beeinträchtigt werden.

\subsubsection{Unterscheidung zwischen Ärzten und Nicht-Ärzten}

Nach dem vorgeschlagenen Wortlaut der Norm würde es bei der grundsätzlichen Differenzierung zwischen Personen aus dem Behandlungsumfeld (nicht nur Ärzte, sondern auch sonstige in $\$ 2$ 291a Abs. 4 SGB V genannte Personen) und sonstigen Personen verbleiben. Dies würde auch bezüglich des Zugriffs auf Behandlungs- oder Forschungsdaten zum Zwecke der Forschung gelten (Frage in Kap. II.2.2.3) und ist aus den in Kapitel II.2.3.4.4 genannten Gründen geboten. 